\author{
Marquette University \\ e-Publications@Marquette
}

\title{
State-Level FDI and Within-Occupation Wage Inequality in The United States
}

\author{
Miao Wang \\ Marquette University, grace.wang@marquette.edu \\ M. C. Sunny Wong \\ University of Houston \\ Hong Zhuang \\ Indiana University South Bend
}

Follow this and additional works at: https://epublications.marquette.edu/econ_fac

Part of the Economics Commons

\section{Recommended Citation}

Wang, Miao; Wong, M. C. Sunny; and Zhuang, Hong, "State-Level FDI and Within-Occupation Wage Inequality in The United States" (2019). Economics Faculty Research and Publications. 601.

https://epublications.marquette.edu/econ_fac/601 


\section{e-Publications@Marquette}

\section{Economics Faculty Research and Publications/College of Business Administration}

This paper is NOT THE PUBLISHED VERSION; but the author's final, peer-reviewed manuscript. The published version may be accessed by following the link in th citation below.

Applied Economics Letters, Vol. 26, No.9x (2019): 770-776. DOI. This article is (C) Taylor \& Francis and permission has been granted for this version to appear in e-Publications@Marquette. Taylor \& Francis does not grant permission for this article to be further copied/distributed or hosted elsewhere without the express permission from Taylor \& Francis.

\section{State-Level FDI and Within-Occupation Wage Inequality in The United States}

\section{Miao Wang}

Department of Economics, College of Business Administration and Graduate School of Management, Marquette University, Milwaukee, WI

\section{C. Sunny Wong} Hobby School of Public Affairs, University of Houston, Houston, TX, Hong Zhuang Judd Leighton School of Business and Economics, Indiana University South Bend, South Bend, IN

\section{Abstract}

Our paper explores the relationship between inward foreign direct investment (FDI) and within-occupation wage inequality at the state level in the U.S. We argue that sectoral FDI may affect different occupations differently, and our study looks at possible heterogeneous effects of sectoral FDI on wage inequality for 22 occupations. Using data over 1999-2007, our results show that state-level manufacturing FDI tends to reduce wage inequality, measured by the ratio of the 90th percentile wage and the 10th percentile wage in an occupation. Manufacturing FDI is significantly associated with less wage inequality in the production occupations and the construction and extraction occupations. In contrast, non-manufacturing FDI is associated with increased withinoccupation wage inequality in the arts, design, entertainment, sports, and media occupations, as well as the 
transportation and material moving occupations. Non-manufacturing FDI is associated with decreased wage inequality in the sales and related occupations.

KEYWORDS: Foreign direct investment, within-occupation income inequality, manufacturing FDI, nonmanufacturing FDI

\section{Introduction}

Studies on the economic growth effect of foreign direct investment (FDI) by multinational corporations (MNCs) abound. The distributional consequences of FDI, however, are largely under-explored. As Figini and Görg (2011) state, 'FDI may bring benefits to the economy in which they locate, but it is unclear whether everyone will benefit to the same extent or indeed whether some will be better off while others will suffer from this'.

In this article, we study the relationship between U.S. inward FDI and within-occupation wage inequality at the state level. While the U.S. is the largest FDI recipient globally, the literature on effects of FDI in the U.S. remains surprisingly small, especially at the subnational level. ${ }^{11} \mathrm{~A}$ few notable exceptions include Blonigen and Figlio (2000), Bode, Nunnenkamp, and Waldkirch (2012), Ford and Rork (2010), Mullen and Williams (2007), and Zhuang (2013). View all notes Further, theoretical predictions regarding the FDI-inequality nexus are not entirely clear. The endowment-driven model suggests that if foreign affiliates are more productive than U.S. domestic firms, FDI raises U.S. demand for skilled-labour relative to unskilled labour as well as the skill premium (Feenstra and Hanson 1997), resulting in greater income inequality. In contrast, the knowledge-capital model argues that MNCs' headquarter services at home are more skilled-labour intensive than their plant operations in foreign hosts (Carr, Markusen, and Maskus 2001). Foreign affiliates' plant-level production in the U.S. is less skilledlabour intensive than the composite rest of the U.S. economy, which often provides headquarter services to other countries (Chintrakarn, Herzer, and Nunnenkamp 2012). Consequently, inward FDI is expected to dampen income inequality in the U.S.

To the best of our knowledge, Chintrakarn, Herzer, and Nunnenkamp (2012) is the only published research on FDI and U.S. state-level income inequality. With data over 1977-2001, the authors find that aggregate FDI reduces the income share of the top $10 \%$ income earners at the state level in the long run, yet the short-run effect of FDI is insignificant.

Complementing Chintrakarn, Herzer, and Nunnenkamp (2012), we focus on sectoral FDI and within-occupation wage inequality for 22 occupations at the state level. U.S. manufacturing receives more inward FDI than any other sector (Saha, Fikri, and Marchio 2014). Arguably, manufacturing and non-manufacturing FDI could have different impacts on income inequality. In addition, although it is possible that FDI can affect all income earners, one would expect that some occupations are more affected by certain foreign investment than other occupations. For instance, manufacturing FDI might affect the wage distribution of production workers rather than those in arts and entertainment.

\section{Model and data}

Our empirical estimation is based on the following model:

$$
\begin{aligned}
& \log \left(\text { Inequality }_{i j t}\right)=\beta_{0}+\beta_{1} \log \left(\text { ManuFDI }_{j t}\right)+\beta_{2} \log \left(\text { non }- \text { ManuFDI }_{j t}\right)+\gamma^{\prime} X_{j t}+ \\
& \alpha_{j}+\tau_{t}+\varepsilon_{i j t}(1)
\end{aligned}
$$


where Inequality $_{i j t}$ is the ratio of the 90 th percentile wage to the 10th percentile wage of occupation $i$, state $j$, and year $t$. Our main variables of interest $M a n u F D I_{j t}$ and non - ManuFDI $I_{j t}$ represent inward FDI in manufacturing and non-manufacturing sectors, respectively. Both are measured by the value of property, plants and equipment of U.S. affiliates of MNCs. The vector $X$ in Equation (1) includes additional control variables that may affect occupational wage distribution (Kim and Sakamoto 2008); $\alpha_{j}$ and $\tau_{t}$ are state and time dummies, respectively.

Other determinants in the vector $X$ include: occupational employment share (empshare ${ }_{i j t}$ ), gross state product $\left(G S P_{j t}\right)$, inflation rate as the percentage change in state GSP deflator (inflation i $_{j t}$ ), unemployment rate $\left(\right.$ urate $\left._{j t}\right)$, share of population over the age of 25 with a college education or above (College $\left.e_{j t}\right)$, total population $\left(\right.$ pop $\left._{j t}\right)$, share of population living in urban areas $\left(\operatorname{urban}_{j t}\right)$, value of exports ( export $\left._{j t}\right)$, and total state and local government expenditure $\left(g \circ v_{j t}\right)$. Controls in vector $\mathrm{X}$ are all measured at the state level except empshare $_{i j t}$, which is an occupation-level variable capturing the employment in occupation $i$, state $j$, and year $t$ as a share of the total occupational employment in the U.S. in year $t$.

Our sample consists of 50 states and Washington D.C. over 1999-2007. We obtain wage data for 22 occupations from the Occupational Employment Statistics Survey, Bureau of Labor Statistics (BLS). FDI data are from the Bureau of Economic Analysis (BEA). Data on other control variables are from the BEA, the BLS, and the Census Bureau. The sample time span is solely determined by data availability. The BLS started to use its current occupational classification system in 1999 and state-level sectoral FDI data are available from the BEA till 2007. Table 1 reports summary statistics of variables. Figure 1 illustrates the 90th/10th wage ratio by occupations.

Table 1. Summary statistics.

\begin{tabular}{|l|l|l|l|l|l|}
\hline Variables & Obs & Mean & Std. dev. & Min & Max \\
\hline $\log$ (inequality) & 9758 & 1.09 & 0.242 & 0.232 & 2.274 \\
\hline $\log$ (manuFDI) & 10,088 & 8.398 & 1.346 & 4.205 & 11.053 \\
\hline $\log$ (non-manuFDI) & 10,088 & 8.681 & 1.125 & 5.38 & 11.318 \\
\hline $\log$ (Export) & 10,088 & 8.606 & 1.387 & 5.255 & 11.838 \\
\hline empshare & 10,052 & 0.02 & 0.024 & 0 & 0.439 \\
\hline $\log$ (GSP) & 10,088 & 11.837 & 1.033 & 9.847 & 14.383 \\
\hline $\log$ (pop) & 10,088 & 1.234 & 1.035 & -0.751 & 3.59 \\
\hline college & 10,088 & 0.173 & 0.038 & 0.104 & 0.332 \\
\hline $\log$ gov) & 10,088 & 10.108 & 1.026 & 8.117 & 12.875 \\
\hline urate & 10,088 & 0.047 & 0.011 & 0.023 & 0.081 \\
\hline inflation & 10,088 & 0.027 & 0.018 & -0.019 & 0.169 \\
\hline urban & 10,088 & 0.728 & 0.15 & 0.376 & 1 \\
\hline
\end{tabular}

Figure 1. Occupational wage inequality over time. 


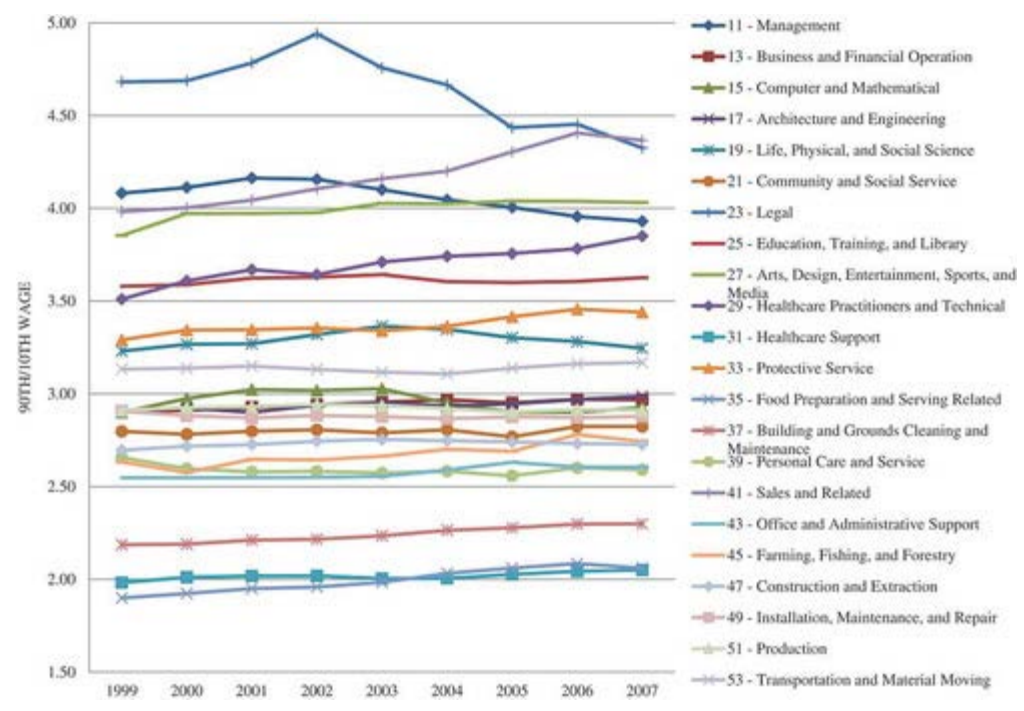

\section{Results and discussions}

We estimate each of the 22 occupational wage regressions using fixed effects and dynamic generalized method of moments (GMM) estimators, and report coefficients on FDI in Table 2. Occupation codes are illustrated in Figure 1. Detailed regression results are available in the Appendix, Tables A1-A2. One might argue that inward FDI may influence within-occupation wage inequality in a state while at the same time state-level wage inequality also affects MNCs location decisions. Therefore, we employ the GMM estimator and instrument the potentially endogenous FDI and lagged dependent variable using their second to eighth lags.

Table 2. Effects of FDI on wage inequality.

\begin{tabular}{|l|l|l|l|l|}
\hline & Manufacturing FDI & Non-manufacturing FDI & & \\
\hline Occupation code & FE & Dynamic GMM & FE & Dynamic GMM \\
\hline 11 & 0.039 & 0.183 & 0.006 & 0.042 \\
\hline & {$[0.023]$} & {$[0.264]$} & {$[0.015]$} & {$[0.198]$} \\
\hline 13 & $-0.023^{* *}$ & -0.022 & $-0.024^{*}$ & 0.039 \\
\hline & {$[0.010]$} & {$[0.079]$} & {$[0.014]$} & {$[0.120]$} \\
\hline 15 & -0.018 & 0.247 & 0.019 & -0.046 \\
\hline & {$[0.020]$} & {$[0.362]$} & {$[0.020]$} & {$[0.364]$} \\
\hline 17 & 0.01 & 0.017 & -0.007 & -0.117 \\
\hline & {$[0.011]$} & {$[0.208]$} & {$[0.016]$} & {$[0.154]$} \\
\hline 19 & -0.033 & -0.126 & -0.015 & 0.029 \\
\hline & {$[0.020]$} & {$[0.212]$} & {$[0.018]$} & {$[0.271]$} \\
\hline 21 & -0.01 & 0 & -0.004 & -0.008 \\
\hline & {$[0.023]$} & {$[0.254]$} & {$[0.016]$} & {$[0.090]$} \\
\hline 23 & 0.032 & 0.26 & 0.038 & 0.25 \\
\hline & {$[0.070]$} & {$[1.783]$} & {$[0.063]$} & {$[1.052]$} \\
\hline 25 & -0.002 & 0.06 & 0.001 & 0 \\
\hline & {$[0.019]$} & {$[0.239]$} & {$[0.017]$} & {$[0.080]$} \\
\hline 27 & 0.013 & -0.040 & $0.035^{*}$ & $0.061^{* *}$ \\
\hline & {$[0.023]$} & {$[0.036]$} & {$[0.019]$} & {$[0.029]$} \\
\hline & & & & \\
\hline & & 0 & & \\
\hline
\end{tabular}




\begin{tabular}{|c|c|c|c|c|}
\hline 29 & 0.032 & 0.171 & 0.031 & 0.186 \\
\hline & {$[0.020]$} & {$[0.785]$} & [0.019] & [0.339] \\
\hline \multirow[t]{2}{*}{31} & 0 & -0.353 & -0.004 & -0.206 \\
\hline & [0.010] & [0.544] & [0.015] & [0.306] \\
\hline \multirow[t]{2}{*}{33} & -0.01 & 0.171 & 0.004 & -0.044 \\
\hline & [0.015] & {$[0.265]$} & {$[0.016]$} & {$[0.189]$} \\
\hline \multirow[t]{2}{*}{35} & -0.008 & 0.087 & 0.002 & -0.071 \\
\hline & {$[0.017]$} & {$[0.175]$} & {$[0.013]$} & {$[0.204]$} \\
\hline \multirow[t]{2}{*}{37} & -0.006 & 0.058 & 0.016 & 0.037 \\
\hline & [0.013] & {$[0.134]$} & {$[0.010]$} & {$[0.103]$} \\
\hline \multirow[t]{2}{*}{39} & -0.012 & 0.061 & 0.013 & -0.06 \\
\hline & {$[0.024]$} & {$[0.293]$} & {$[0.023]$} & {$[0.307]$} \\
\hline \multirow[t]{2}{*}{41} & -0.02 & 0.021 & $-0.028 *$ & $-0.029 * *$ \\
\hline & {$[0.022]$} & {$[0.020]$} & [0.015] & {$[0.012]$} \\
\hline \multirow[t]{2}{*}{43} & -0.009 & 0.064 & 0.006 & 0.104 \\
\hline & [0.006] & [0.165] & [0.005] & [0.166] \\
\hline \multirow[t]{2}{*}{45} & -0.077 & -1.085 & 0.028 & -0.166 \\
\hline & {$[0.049]$} & [1.212] & {$[0.032]$} & {$[0.375]$} \\
\hline \multirow[t]{2}{*}{47} & $-0.026 *$ & $-0.039 *$ & -0.004 & 0.003 \\
\hline & [0.015] & {$[0.023]$} & [0.009] & {$[0.026]$} \\
\hline \multirow[t]{2}{*}{49} & -0.002 & -0.089 & $-0.022^{*}$ & -0.009 \\
\hline & {$[0.016]$} & {$[0.181]$} & {$[0.012]$} & [0.152] \\
\hline \multirow[t]{2}{*}{51} & $-0.027^{*}$ & $-0.026^{*}$ & -0.009 & $0.035 *$ \\
\hline & [0.014] & [0.014] & [0.010] & {$[0.020]$} \\
\hline \multirow[t]{2}{*}{53} & -0.019 & -0.029 & $0.021^{* * *}$ & $0.038^{* *}$ \\
\hline & [0.017] & {$[0.031]$} & {$[0.007]$} & {$[0.014]$} \\
\hline
\end{tabular}

Table 2 shows considerable heterogeneity regarding the effect of state-level FDI on wage inequality. Fixed effects results show that manufacturing FDI has a significantly negative effect on the wage gap in 3 of the 22 occupation regressions, namely, business and financial operations occupations, construction and extraction occupations, and production occupations. The coefficients on manufacturing FDI in other regressions are often negative, but not significant at conventional levels. In comparison, the coefficient on non-manufacturing FDI is negative and significant in three regressions, including business and financial operations occupations, sales and related occupations, and installation, maintenance, and repair occupations. The coefficient on nonmanufacturing FDI is positive and significant for two occupations - arts, design, entertainment, sports, and media occupations and transportation and material moving occupations.

The GMM results are, in general, similar to the fixed effects results. Manufacturing FDI has a negative and significant effect in two occupations. Non-manufacturing FDI has a positive effect on wage inequality in 3 of the 22 regressions and a negative effect in one regression.

For ease of comparison, we highlight in bold the coefficient on FDI that is least sensitive to model specifications and consistent across both estimators, in terms of the sign of the coefficient and its statistical significance. The 
effects of manufacturing FDI are robust and consistent in construction and extraction occupations (occupation code: 47 ) and production occupations (51). In both occupations, manufacturing FDI tends to decrease the gap between the 90th and 10th percentile occupational wages. Results regarding the effects of non-manufacturing FDI that are robust and consistent across both estimators are in arts, design, entertainment, sports and media occupations (27); sales and related occupations (41); and transportation and material moving occupations (53). As manufacturing and non-manufacturing FDI tends to affect within-occupation wage inequality differently in different occupations, our findings might provide a possible explanation to the insignificant short-run effect of total FDI on state-level income inequality in Chintrakarn, Herzer, and Nunnenkamp (2012).

Since our wage inequality and FDI variables enter the regressions in log form, the estimated coefficient on FDI represents elasticity. The dynamic GMM estimates suggest that each year a $1 \%$ rise in manufacturing FDI in a state is associated with a $0.039 \%$ decrease in the gap between the 90th and 10th percentile wage in construction and extraction occupations, and a $0.026 \%$ decrease in that same gap in production occupations, ceteris paribus. For the two occupations for which non-manufacturing FDI is shown to have a robustly positive effect on wage inequality, a $1 \%$ rise in non-manufacturing FDI is associated with a $0.061 \%$ increase in the wage gap in arts occupations and a $0.038 \%$ increase in the wage gap in the transportation and material moving occupations. In sales occupations, a $1 \%$ rise in non-manufacturing FDI is associated with a $0.029 \%$ decrease in the wage gap according to the GMM results. These coefficients are small but economically meaningful, considering the slow-moving nature of wage gaps in these occupations.

The inequality-dampening effects of manufacturing FDI in production occupations are consistent with theoretical predictions from the knowledge-capital model. These results are important, as about $18.5 \%$ of manufacturing jobs in the U.S. are supported by FDI (Saha, Fikri, and Marchio 2014) and 63.9\% of manufacturing FDI employment could be in production and related occupations (Bureau of Labor Statistics 1993). The inequality-increasing effects of non-manufacturing FDI in some occupations are interesting, especially since the share of non-manufacturing FDI in the U.S. has been rising over time. An important direction for future research would be to look into the distributions of occupations in foreign-owned manufacturing and non-manufacturing establishments and further explore the underlying reasons for the heterogeneous effects of FDI on income distributions. Such future research may depend on more recent data being made available to the general public.

\section{Disclosure statement}

No potential conflict of interest was reported by the authors. 


\section{Appendix}

Table A1. Fixed effects estimation of within-occupation wage inequality.

\begin{tabular}{|c|c|c|c|c|c|c|c|c|c|c|c|}
\hline- & -1 & -2 & -3 & -4 & -5 & -6 & -7 & -8 & -9 & -10 & -11 \\
\hline Occupation & 11 & 13 & 15 & 17 & 19 & 21 & 23 & 25 & 27 & 29 & 31 \\
\hline $\log ($ manuFDI) & 0.039 & $-0.023^{* *}$ & -0.018 & 0.01 & -0.033 & -0.010 & 0.032 & -0.002 & 0.013 & 0.032 & -0.000 \\
\hline- & [0.023] & [0.010] & [0.020] & [0.011] & [0.020] & [0.023] & [0.070] & [0.019] & [0.023] & {$[0.020]$} & [0.010] \\
\hline $\log ($ non-manuFDI) & 0.006 & $-0.024^{*}$ & 0.019 & -0.007 & -0.015 & -0.004 & 0.038 & 0.001 & $0.035^{*}$ & 0.031 & -0.004 \\
\hline- & [0.015] & {$[0.014]$} & {$[0.020]$} & {$[0.016]$} & [0.018] & {$[0.016]$} & [0.063] & [0.017] & [0.019] & [0.019] & [0.015] \\
\hline $\log$ (Export) & 0.008 & 0.022 & -0.004 & $-0.032 *$ & 0.015 & 0.009 & 0.052 & -0.020 & 0.05 & $-0.078 * *$ & $-0.036^{* *}$ \\
\hline- & [0.029] & [0.014] & {$[0.026]$} & [0.018] & [0.023] & [0.017] & [0.073] & [0.029] & [0.035] & [0.033] & {$[0.017]$} \\
\hline empshare & $10.419 * * *$ & -0.725 & 1.478 & 0.551 & $7.740 * *$ & 1.34 & 5.35 & 1.49 & 3.827 & 0.874 & $-3.394^{* *}$ \\
\hline- & {$[3.422]$} & {$[2.318]$} & [2.190] & [2.663] & [3.247] & [2.683] & [5.175] & [1.527] & {$[2.720]$} & [3.306] & {$[1.404]$} \\
\hline $\log (G S P)$ & -0.152 & -0.049 & -0.025 & 0.095 & -0.057 & -0.123 & 0.16 & 0.068 & -0.191 & 0.176 & -0.080 \\
\hline- & [0.144] & [0.117] & [0.145] & {$[0.137]$} & {$[0.233]$} & {$[0.160]$} & [0.397] & {$[0.125]$} & [0.160] & {$[0.240]$} & {$[0.112]$} \\
\hline $\log ($ pop) & 0.238 & $0.423^{* *}$ & -0.077 & -0.023 & 0.279 & $0.325^{*}$ & 0.15 & -0.280 & -0.035 & 0.115 & -0.046 \\
\hline- & [0.279] & [0.159] & {$[0.282]$} & {$[0.204]$} & [0.263] & {$[0.176]$} & {$[0.420]$} & [0.233] & [0.289] & {$[0.410]$} & {$[0.218]$} \\
\hline college & 0.372 & $-0.426^{* *}$ & -0.298 & 0.159 & 0.176 & -0.153 & 0.072 & 0.395 & 0.297 & 0.033 & $-0.515^{*}$ \\
\hline- & {$[0.347]$} & {$[0.206]$} & {$[0.263]$} & {$[0.214]$} & [0.409] & [0.318] & [0.940] & {$[0.297]$} & {$[0.341]$} & {$[0.451]$} & {$[0.260]$} \\
\hline $\log$ (gov) & -0.004 & -0.050 & -0.113 & 0.006 & -0.092 & -0.118 & $0.516^{*}$ & $-0.249 * *$ & 0.034 & -0.217 & 0.011 \\
\hline- & [0.136] & [0.087] & {$[0.214]$} & {$[0.100]$} & [0.130] & [0.091] & [0.279] & {$[0.124]$} & [0.135] & [0.184] & [0.111] \\
\hline urate & 0.195 & 0.083 & -0.751 & 0.214 & 0.719 & $-0.968^{*}$ & -1.483 & -1.104 & 0.122 & -1.031 & 0.284 \\
\hline- & [0.996] & [0.501] & [0.819] & [0.781] & [0.817] & {$[0.564]$} & [2.151] & {$[0.667]$} & [0.935] & [0.966] & {$[0.864]$} \\
\hline inflation & -0.196 & $-0.246 * *$ & -0.409 & -0.003 & -0.075 & 0.283 & $-0.954 * *$ & $0.330^{* *}$ & 0.154 & 0.071 & 0.098 \\
\hline- & {$[0.232]$} & [0.109] & [0.453] & {$[0.172]$} & {$[0.164]$} & {$[0.206]$} & [0.432] & {$[0.144]$} & [0.197] & [0.208] & {$[0.138]$} \\
\hline urban & 1.478 & -1.247 & -0.417 & 0.695 & -0.486 & -0.335 & -0.554 & -0.400 & -0.729 & 0.256 & 1.342 \\
\hline- & [1.421] & {$[0.766]$} & [1.345] & [0.979] & [0.973] & {$[0.815]$} & {$[2.214]$} & {$[0.898]$} & [1.263] & [1.279] & {$[0.901]$} \\
\hline Constant & 1.323 & 2.831 & 2.929 & -0.384 & 2.83 & $3.564^{*}$ & -6.006 & $3.667^{* *}$ & 2.854 & 1.133 & 1.081 \\
\hline- & {$[2.238]$} & [1.690] & [2.636] & [1.916] & [2.727] & [1.863] & [6.506] & [1.808] & [2.372] & [2.997] & {$[2.016]$} \\
\hline Observations & 306 & 458 & 459 & 457 & 458 & 455 & 290 & 451 & 458 & 458 & 458 \\
\hline R-squared & 0.2 & 0.14 & 0.12 & 0.1 & 0.09 & 0.06 & 0.13 & 0.11 & 0.14 & 0.2 & 0.15 \\
\hline
\end{tabular}


Table A1. (Continued).

\begin{tabular}{|c|c|c|c|c|c|c|c|c|c|c|c|}
\hline- & -12 & -13 & -14 & -15 & -16 & -17 & -18 & -19 & -20 & -21 & -22 \\
\hline Occupation & 33 & 35 & 37 & 39 & 41 & 43 & 45 & 47 & 49 & 51 & 53 \\
\hline $\log$ (manuFDI) & -0.010 & -0.008 & -0.006 & -0.012 & -0.020 & -0.009 & -0.077 & $-0.026^{*}$ & -0.002 & $-0.027^{*}$ & -0.019 \\
\hline- & [0.015] & {$[0.017]$} & {$[0.013]$} & {$[0.024]$} & {$[0.022]$} & {$[0.006]$} & [0.049] & [0.015] & {$[0.016]$} & {$[0.014]$} & [0.017] \\
\hline $\log ($ non-manuFDI) & 0.004 & 0.002 & 0.016 & 0.013 & $-0.028^{*}$ & 0.006 & 0.028 & -0.004 & $-0.022^{*}$ & -0.009 & $0.021^{* * *}$ \\
\hline- & {$[0.016]$} & {$[0.013]$} & {$[0.010]$} & {$[0.023]$} & {$[0.015]$} & {$[0.005]$} & {$[0.032]$} & [0.009] & {$[0.012]$} & {$[0.010]$} & {$[0.007]$} \\
\hline $\log$ (Export) & 0.027 & 0.011 & 0.021 & 0.022 & $0.042^{* *}$ & $0.016^{* *}$ & 0.027 & $0.017^{*}$ & -0.020 & 0.006 & -0.023 \\
\hline- & {$[0.017]$} & {$[0.016]$} & [0.017] & {$[0.036]$} & [0.017] & {$[0.007]$} & [0.039] & {$[0.010]$} & {$[0.016]$} & {$[0.013]$} & {$[0.016]$} \\
\hline empshare & 1.428 & -1.030 & -3.013 & $-13.133^{* * *}$ & $4.388^{*}$ & $6.983^{* *}$ & $-2.783^{*}$ & -1.691 & 5.413* & 0.057 & $4.042^{* * *}$ \\
\hline- & [3.435] & [5.439] & [5.033] & [3.741] & {$[2.405]$} & [3.007] & [1.422] & [1.258] & {$[2.895]$} & [1.357] & [1.468] \\
\hline $\log (G S P)$ & 0.047 & -0.182 & -0.120 & 0.292 & -0.226 & $-0.130 * *$ & $-0.488^{*}$ & 0.112 & $-0.159 *$ & -0.152 & $-0.271 * * *$ \\
\hline- & [0.141] & [0.115] & [0.131] & {$[0.253]$} & [0.143] & [0.063] & {$[0.249]$} & [0.094] & [0.094] & {$[0.097]$} & {$[0.080]$} \\
\hline $\log ($ pop) & -0.136 & 0.113 & -0.166 & -0.369 & $-0.415^{* *}$ & $0.195^{* *}$ & $0.542^{*}$ & -0.080 & 0.037 & 0.248 & -0.075 \\
\hline- & {$[0.242]$} & {$[0.207]$} & [0.176] & {$[0.381]$} & {$[0.187]$} & [0.095] & [0.282] & [0.122] & [0.110] & {$[0.154]$} & [0.144] \\
\hline college & -0.291 & $-0.726 * *$ & -0.261 & -0.185 & $-0.624^{* *}$ & -0.145 & $-0.993^{*}$ & 0.306 & 0.013 & 0.226 & 0.121 \\
\hline- & {$[0.306]$} & {$[0.285]$} & {$[0.222]$} & {$[0.423]$} & {$[0.308]$} & [0.131] & [0.575] & [0.191] & {$[0.230]$} & {$[0.186]$} & {$[0.166]$} \\
\hline $\log$ gov) & -0.004 & $0.276^{* *}$ & $0.190^{* *}$ & $0.456^{* *}$ & 0.163 & -0.050 & 0.288 & 0.035 & $-0.149 * *$ & -0.022 & $0.159 *$ \\
\hline- & {$[0.133]$} & {$[0.124]$} & {$[0.094]$} & [0.183] & {$[0.100]$} & {$[0.074]$} & {$[0.178]$} & {$[0.073]$} & {$[0.066]$} & [0.079] & {$[0.080]$} \\
\hline urate & $-0.848^{*}$ & -0.796 & $-1.292^{* *}$ & -0.120 & -0.239 & 0.066 & -1.312 & 0.213 & -0.444 & -0.566 & $-0.862 * *$ \\
\hline- & {$[0.490]$} & {$[0.552]$} & {$[0.504]$} & {$[1.642]$} & {$[0.673]$} & {$[0.323]$} & [1.311] & {$[0.463]$} & {$[0.452]$} & {$[0.412]$} & {$[0.347]$} \\
\hline inflation & 0.092 & 0.073 & $0.320^{* *}$ & $1.035^{* * *}$ & $0.197^{*}$ & $-0.138^{* *}$ & 0.47 & 0.004 & $-0.294^{*}$ & -0.202 & 0.178 \\
\hline- & [0.099] & {$[0.117]$} & {$[0.127]$} & {$[0.269]$} & [0.104] & {$[0.065]$} & {$[0.466]$} & [0.110] & {$[0.147]$} & [0.159] & {$[0.116]$} \\
\hline urban & $1.580 *$ & $2.564 * * *$ & $3.161 * * *$ & 2.498 & $2.977^{* * *}$ & 0.536 & -0.477 & 0.415 & $1.912 * *$ & $1.619 * *$ & $1.751^{* *}$ \\
\hline- & {$[0.921]$} & {$[0.862]$} & [0.792] & [1.631] & [0.931] & [0.389] & [1.556] & {$[0.860]$} & {$[0.785]$} & {$[0.722]$} & {$[0.864]$} \\
\hline Constant & -0.437 & -1.797 & -1.875 & $-8.243 * *$ & 0.853 & $2.116^{* *}$ & 3.978 & -0.790 & $3.263 * *$ & 1.835 & 1.686 \\
\hline- & [2.333] & [1.984] & [1.751] & [3.872] & [2.083] & [0.850] & [3.176] & [1.385] & {$[1.259]$} & [1.312] & [1.683] \\
\hline Observations & 459 & 459 & 458 & 454 & 454 & 459 & 443 & 456 & 459 & 457 & 459 \\
\hline R-squared & 0.17 & 0.46 & 0.39 & 0.17 & 0.55 & 0.35 & 0.18 & 0.1 & 0.18 & 0.15 & 0.17 \\
\hline
\end{tabular}


Table A2. Dynamic GMM estimation of within-occupation wage inequality.

\begin{tabular}{|c|c|c|c|c|c|c|c|c|c|c|c|}
\hline & -1 & -2 & -3 & -4 & -5 & -6 & -7 & -8 & -9 & -10 & -11 \\
\hline Occupation & 11 & 13 & 15 & 17 & 19 & 21 & 23 & 25 & 27 & 29 & 31 \\
\hline \multirow[t]{2}{*}{$\log ($ manuFDI) } & 0.183 & -0.022 & 0.247 & 0.017 & -0.126 & -0.000 & 0.26 & 0.06 & -0.040 & 0.171 & -0.353 \\
\hline & {$[0.264]$} & [0.079] & [0.362] & [0.208] & [0.212] & {$[0.254]$} & [1.783] & [0.239] & {$[0.036]$} & [0.785] & {$[0.544]$} \\
\hline \multirow[t]{2}{*}{$\log ($ non-manuFDI) } & 0.042 & 0.039 & -0.046 & -0.117 & 0.029 & -0.008 & 0.25 & 0 & $0.061 * *$ & 0.186 & -0.206 \\
\hline & [0.198] & {$[0.120]$} & {$[0.364]$} & {$[0.154]$} & {$[0.271]$} & {$[0.090]$} & {$[1.052]$} & {$[0.080]$} & [0.029] & [0.339] & {$[0.306]$} \\
\hline \multirow[t]{2}{*}{$\log$ (Export) } & -0.130 & -0.077 & -0.172 & -0.000 & -0.034 & 0.038 & 0.214 & -0.044 & 0.016 & -0.171 & 0.04 \\
\hline & [0.141] & [0.110] & [0.308] & [0.068] & [0.106] & [0.141] & [0.456] & {$[0.236]$} & [0.025] & [0.455] & [0.178] \\
\hline \multirow[t]{2}{*}{ empshare } & 4.729 & 40.538 & 20.014 & 0.478 & 21.402 & 18.197 & 159.084 & 23.422 & 0.204 & 40.402 & 31.757 \\
\hline & [60.513] & [49.172] & [105.698] & [36.905] & [34.449] & [25.087] & {$[805.080]$} & [39.528] & {$[0.472]$} & [136.354] & [72.769] \\
\hline \multirow[t]{2}{*}{$\log (G S P)$} & -0.116 & 0.971 & 1.794 & -0.029 & -0.533 & -0.575 & 4.268 & 2.037 & -0.007 & -0.768 & -0.044 \\
\hline & [2.208] & [1.577] & [4.767] & {$[0.722]$} & {$[2.444]$} & [2.275] & [5.945] & [5.884] & [0.073] & [2.186] & [1.289] \\
\hline \multirow[t]{2}{*}{$\log (p o p)$} & 1.065 & 0.156 & 1.089 & -0.447 & 0.169 & -0.377 & -5.353 & -1.272 & $0.123^{*}$ & 0.936 & -1.033 \\
\hline & [1.977] & [1.646] & [2.709] & {$[1.073]$} & [1.299] & [1.604] & {$[7.730]$} & [3.759] & {$[0.071]$} & [5.637] & [1.969] \\
\hline \multirow[t]{2}{*}{ college } & 3.743 & 2.612 & 4.959 & -0.425 & 1.556 & -0.454 & 0.826 & -2.242 & -0.093 & -9.954 & 2.653 \\
\hline & [8.228] & [2.590] & [14.477] & [2.659] & [2.859] & [6.335] & [6.873] & [14.108] & {$[0.307]$} & [8.936] & [3.586] \\
\hline \multirow[t]{2}{*}{ log(gov) } & -1.273 & -1.079 & -3.300 & -0.018 & 1.046 & 0.733 & 1.817 & -1.100 & -0.098 & -1.565 & 1.095 \\
\hline & [3.096] & [1.281] & [3.436] & [0.692] & [3.118] & [4.141] & [7.440] & [2.050] & [0.106] & [8.955] & [1.788] \\
\hline \multirow[t]{2}{*}{ urate } & 6.575 & 7.259 & 17.023 & 3.54 & 2.515 & -2.479 & 0.312 & -3.864 & -1.204 & -22.360 & 10.303 \\
\hline & [14.785] & {$[6.607]$} & {$[20.676]$} & [7.317] & [5.639] & {$[11.767]$} & [29.697] & {$[32.686]$} & {$[0.790]$} & {$[22.490]$} & [10.952] \\
\hline \multirow[t]{2}{*}{ inflation } & 1.845 & 0.075 & 1.101 & -0.062 & -1.854 & -2.303 & -0.700 & -2.575 & -0.052 & -1.163 & -0.276 \\
\hline & [6.319] & [1.449] & [5.056] & [1.854] & [1.970] & [2.736] & [3.996] & [8.224] & [0.132] & [2.294] & [1.376] \\
\hline \multirow[t]{2}{*}{ urban } & 1.165 & -3.791 & -0.141 & 1.157 & -2.915 & -0.239 & -8.840 & -0.949 & -0.104 & 4.892 & -4.881 \\
\hline & [2.925] & [2.859] & [8.951] & [3.657] & [4.231] & [2.186] & [27.394] & [2.458] & [0.150] & [16.768] & [6.959] \\
\hline \multirow[t]{2}{*}{ Lagged 90th/10th wage ratio } & 0.626 & 0.506 & 0.587 & -0.324 & 0.111 & 0.491 & -0.514 & -0.035 & $0.515^{* *}$ & -0.106 & 0.131 \\
\hline & {$[0.796]$} & {$[0.547]$} & {$[0.457]$} & {$[0.400]$} & {$[0.479]$} & {$[0.666]$} & {$[1.245]$} & {$[0.736]$} & {$[0.205]$} & {$[2.024]$} & {$[0.927]$} \\
\hline Observations & 258 & 407 & 408 & 406 & 407 & 403 & 238 & 395 & 407 & 406 & 407 \\
\hline Hansen p-value & 0.869 & 0.569 & 0.319 & 0.561 & 0.338 & 0.315 & 0.747 & 0.487 & 0.189 & 0.679 & 0.599 \\
\hline$A R(2) p$-value & 0.626 & 0.783 & 0.569 & 0.739 & 0.398 & 0.729 & 0.709 & 0.719 & 0.195 & 0.264 & 0.791 \\
\hline
\end{tabular}


Table A2. (Continued).

\begin{tabular}{|c|c|c|c|c|c|c|c|c|c|c|c|}
\hline & -12 & -13 & -14 & -15 & -16 & -17 & -18 & -19 & -20 & -21 & -22 \\
\hline Occupation & 33 & 35 & 37 & 39 & 41 & 43 & 45 & 47 & 49 & 51 & 53 \\
\hline \multirow[t]{2}{*}{$\log ($ manuFDI) } & 0.171 & 0.087 & 0.058 & 0.061 & 0.021 & 0.064 & -1.085 & $-0.039 *$ & -0.089 & $-0.026^{*}$ & -0.029 \\
\hline & {$[0.265]$} & [0.175] & [0.134] & {$[0.293]$} & {$[0.020]$} & {$[0.165]$} & [1.212] & [0.023] & [0.181] & [0.014] & {$[0.031]$} \\
\hline \multirow[t]{2}{*}{$\log ($ non-manuFDI) } & -0.044 & -0.071 & 0.037 & -0.060 & $-0.029 * *$ & 0.104 & -0.166 & 0.003 & -0.009 & $0.035^{*}$ & $0.038^{* *}$ \\
\hline & [0.189] & {$[0.204]$} & {$[0.103]$} & {$[0.307]$} & {$[0.012]$} & {$[0.166]$} & {$[0.375]$} & {$[0.026]$} & {$[0.152]$} & {$[0.020]$} & {$[0.014]$} \\
\hline \multirow[t]{2}{*}{$\log$ (Export) } & -0.001 & -0.024 & -0.034 & 0.175 & -0.019 & 0.002 & 0.033 & 0.02 & -0.127 & 0.008 & 0.027 \\
\hline & {$[0.165]$} & {$[0.087]$} & {$[0.064]$} & {$[0.174]$} & {$[0.014]$} & [0.132] & [0.683] & {$[0.014]$} & [0.081] & {$[0.027]$} & {$[0.020]$} \\
\hline \multirow[t]{2}{*}{ empshare } & -10.165 & 7.065 & 20.017 & -47.580 & 0.134 & 15.238 & -14.375 & $-0.598 *$ & 27.199 & -0.891 & -0.035 \\
\hline & {$[53.520]$} & [18.561] & [35.665] & [66.421] & {$[0.328]$} & {$[60.777]$} & [17.213] & {$[0.326]$} & [80.599] & {$[1.354]$} & {$[0.312]$} \\
\hline \multirow[t]{2}{*}{$\log (G S P)$} & -0.604 & 1.208 & -0.459 & 2.503 & 0.036 & -0.001 & -0.600 & -0.029 & -1.307 & 0.045 & 0.015 \\
\hline & [1.382] & [1.349] & [0.664] & [1.849] & [0.039] & {$[1.266]$} & [5.836] & {$[0.092]$} & [1.442] & {$[0.067]$} & [0.049] \\
\hline \multirow[t]{2}{*}{$\log ($ pop) } & 0.93 & -1.108 & -0.162 & -1.634 & $-0.046^{*}$ & 0.668 & 0.054 & -0.035 & 0.915 & -0.049 & $0.085^{* * *}$ \\
\hline & [1.614] & {$[0.850]$} & [0.662] & [1.704] & {$[0.023]$} & [1.485] & [7.476] & [0.065] & [0.864] & {$[0.106]$} & {$[0.029]$} \\
\hline \multirow[t]{2}{*}{ college } & 4.563 & $-4.061 * *$ & -2.111 & -0.380 & 0.008 & -2.443 & -1.938 & -0.309 & 3.297 & -0.288 & 0.046 \\
\hline & [8.176] & [1.937] & {$[2.072]$} & [3.409] & [0.151] & {$[4.704]$} & [11.934] & {$[0.450]$} & [3.778] & {$[0.286]$} & {$[0.248]$} \\
\hline \multirow[t]{2}{*}{$\log ($ gov $)$} & -0.728 & -0.193 & 0.393 & 0.073 & 0.036 & -1.461 & 2.404 & 0.109 & 0.162 & 0.021 & $-0.126^{* *}$ \\
\hline & {$[2.096]$} & {$[1.546]$} & [1.219] & {$[2.307]$} & {$[0.040]$} & [1.462] & [5.119] & {$[0.152]$} & [1.395] & [0.099] & {$[0.052]$} \\
\hline \multirow[t]{2}{*}{ urate } & 4.578 & 2.162 & -1.315 & 13.784 & 0.527 & -2.712 & -12.955 & 0.405 & 1.259 & -1.568 & -0.320 \\
\hline & [10.179] & {$[6.656]$} & [3.010] & {$[8.426]$} & {$[0.478]$} & {$[8.908]$} & [35.041] & [1.129] & [7.933] & [1.284] & [0.499] \\
\hline \multirow[t]{2}{*}{ inflation } & 0.972 & -0.817 & 0.21 & 1.632 & -0.077 & -1.062 & 6.883 & -0.004 & -0.448 & -0.434 & 0.043 \\
\hline & {$[2.236]$} & {$[0.972]$} & {$[0.601]$} & [1.828] & [0.139] & [1.380] & [15.463] & {$[0.147]$} & [1.650] & {$[0.282]$} & [0.177] \\
\hline \multirow[t]{2}{*}{ urban } & 3.374 & -0.767 & -0.198 & -2.324 & 0.058 & 2.556 & -6.011 & 0.04 & -0.662 & -0.175 & -0.116 \\
\hline & [6.339] & {$[2.630]$} & [3.335] & [3.773] & {$[0.065]$} & [5.263] & [6.908] & {$[0.121]$} & [4.380] & {$[0.123]$} & {$[0.088]$} \\
\hline \multirow[t]{2}{*}{ Lagged 90th/10th wage ratio } & 0.187 & 0.347 & 0.169 & 0.585 & $0.901^{* * *}$ & -0.372 & -0.488 & $0.637^{* *}$ & --0.171 & $0.884 * * *$ & $1.034^{* * *}$ \\
\hline & "[0.883]" & {$[0.667]$} & [0.352] & {$[0.506]$} & {$[0.100]$} & [1.163] & {$[1.983]$} & {$[0.296]$} & {$[0.618]$} & {$[0.081]$} & {$[0.139]$} \\
\hline Observations & 408 & 408 & 406 & 402 & 402 & 408 & 392 & 405 & 408 & 405 & 408 \\
\hline Hansen p-value & 0.29 & 0.65 & 0.0644 & 0.408 & 0.132 & 0.431 & 0.684 & 0.14 & 0.482 & 0.477 & 0.261 \\
\hline$A R(2) p$-value & 0.755 & 0.164 & 0.594 & 0.807 & 0.201 & 0.752 & 0.624 & 0.896 & 0.767 & 0.145 & 0.983 \\
\hline
\end{tabular}


Notes

1 A few notable exceptions include Blonigen and Figlio (2000), Bode, Nunnenkamp, and Waldkirch (2012), Ford and Rork (2010), Mullen and Williams (2007), and Zhuang (2013).

\section{References}

Blonigen, B., and D. Figlio. 2000. "The Effects of Foreign Direct Investment on Local Communities." Journal of Urban Economics 48 (2): 338-363. doi:10.1006/juec.2000.2170.

Bode, E., P. Nunnenkamp, and A. Waldkirch. 2012. "Spatial Effects of Foreign Direct Investment in US States." Canadian Journal of Economics 45 (1): 16-40. doi:10.1111/j.1540-5982.2011.01685.x.

Bureau of Labor Statistics (1993). New Research on Occupations in Foreign-Owned Manufacturing Establishments in the United States. Available at: https://www.bls.gov/news.release/fome.toc.htm

Carr, D., J. Markusen, and K. Maskus. 2001. "Estimating the Knowledge-Capital Model of the Multinational Enterprise." American Economic Review 91 (3): 693-708. doi:10.1257/aer.91.3.693.

Chintrakarn, P., D. Herzer, and P. Nunnenkamp. 2012. "FDI and Income Inequality: Evidence from a Panel of US States." Economic Inquiry 50 (3): 788-801. doi:10.1111/ecin.2012.50.issue-3.

Feenstra, R., and G. Hanson. 1997. “Foreign Direct Investment and Relative Wages: Evidence from Mexico's Maquiladoras." Journal of International Economics 42: 371-3931. doi:10.1016/S0022-1996(96)01475-4.

Figini, P., and H. Görg. 2011. "Does Foreign Direct Investment Affect Wage Inequality? An Empirical Investigation." World Economy 34 (9): 1455-1475. doi:10.1111/j.1467-9701.2011.01397.x.

Ford, T., and J. Rork. 2010. "Why Buy What You Can Get for Free? The Effect of Foreign Direct Investment on State Patent Rates." Journal of Urban Economics 68 (1): 72-81. doi:10.1016/j.jue.2010.03.001.

Kim, C., and A. Sakamoto. 2008. "The Rise of Intra-Occupational Wage Inequality in the U.S.,1983 to 2002." American Sociological Review 73 (1): 129-157. doi:10.1177/000312240807300107.

Mullen, J., and M. Williams. 2007. "Foreign Direct Investment and Regional Productivity Spillovers in US." Review of Urban \& Regional Development Studies 19 (3): 185-196. doi:10.1111/j.1467-940X.2007.00137.x.

Saha, D., K. Fikri, and N. Marchio. 2014. FDI in U.S. Metro Areas: The Geography of Jobs in Foreign-Owned Establishments. Global Cities Initiative. Brookings Institute: Washington, D.C.

Zhuang, H. 2013. "The Effect of FDI on Local Educational Expenditures: Evidence from the United States." Bulletin of Economic Research 65 (3): 203-224. doi:10.1111/j.1467-8586.2011.00404.x. 